\title{
Estudio clínico y epidemiológico de la criptococosis en Colombia: resultados de nueve años de la encuesta nacional, 1997-2005
}

\author{
Jairo Lizarazo ${ }^{1}$, Melva Linares ${ }^{2}$, Catalina de Bedout ${ }^{3}$, Ángela Restrepo ${ }^{3}$, \\ Clara Inés Agudelo ${ }^{2}$, Elizabeth Castañeda ${ }^{2}$ y Grupo Colombiano para el Estudio \\ de la Criptococosis ${ }^{4}$ \\ ${ }^{1}$ Hospital Universitario Erasmo Meoz, Cúcuta, Colombia \\ ${ }^{2}$ Grupo de Microbiología, Instituto Nacional de Salud, Bogotá D.C., Colombia \\ ${ }^{3}$ Corporación para Investigaciones Biológicas, Medellín, Colombia.
}

Introducción. Con el fin de conocer la situación de la criptococosis en Colombia se ha llevado a cabo una encuesta nacional. El presente estudio analiza la información recogida de 1997 a 2005.

Materiales y métodos. Se utilizó el formato de encuesta de la Confederación Europea de Micología Médica.

Resultados. Se recibieron 931 encuestas de 76 centros. Se identificaron 891 (95,7\%) casos de neurocriptococosis, 27 (2,9\%) de formas pulmonares, cinco (0,5\%) de lesiones cutáneas, dos $(0,2 \%)$ de ganglionares, dos $(0,2 \%)$ de úlceras orofaríngeas y un caso $(0,1 \%)$ de cada una de las siguientes formas clínicas: peritonitis, lesión hepática, celulitis de los miembros inferiores e infección de las vías urinarias. El 82,7\% fueron hombres con predominio de los adultos jóvenes. Sin embargo, se reportaron $25(2,7 \%)$ niños. La infección por el virus de la inmunodeficiencia humana se encontró en el $78,1 \%$ de los casos. La incidencia promedio anual de criptococosis en la población general fue de 2,4 casos $\times 10^{6}$, pero en los pacientes con sida se elevó a 3,0 casos $\times 10^{3}$. Las manifestaciones clínicas más frecuentes fueron: cefalea $(85,2 \%)$, náuseas y vómito $(59,1 \%)$, fiebre $(59 \%)$, cambios mentales $(46,2 \%)$ y signos meníngeos (33,4\%). El examen directo de líquido cefalorraquídeo fue positivo en el 92,8\% de los casos y el hongo se recuperó en el 90,3\%. El antígeno capsular tuvo una positividad del $98,9 \%$ en el líquido cefalorraquídeo y del 93,7\% en el suero. De 788 aislamientos remitidos, 95,9\% fueron Cryptococcus neoformans var. grubii serotipo A, 0,3\%, var. neoformans serotipo D, 3,3\%, C. gattii serotipo B y $0,5 \%$, C. gattii serotipo C. La mayoría de los pacientes fueron tratados inicialmente con anfotericina $\mathrm{B}$.

Conclusión: La criptococosis es una enfermedad cuya incidencia aumentó con la pandemia del VIH y constituye un marcador centinela de sida en Colombia.

Palabras clave: criptococosis, Cryptococcus neoformans, síndrome de inmunodeficiencia adquirida, morbilidad, mortalidad, Colombia.

Results of nine years of the clinical and epidemiological survey on cryptococcosis in Colombia, 1997-2005

Introduction. A national survey on cryptococcosis has been conducted in Colombia since 1997. The survey data recorded over a 9-year period, 1997 to 2005, was summarized.

Materials and methods. The format provided by the European Confederation of Medical Mycology was adapted with the correspondent permission.

Results. Over the 9 year period, 931 surveys were received from 76 centers. The associated disease syndromes were as follows: 891 (95.7\%) were neurocryptococosis cases, 27 (2.9\%) pulmonary disease, $5(0.5 \%)$ cutaneous lesions, $2(0.2 \%)$ ganglionar forms, $2(0.2 \%)$ oropharyngeal lesions and one case $(0.1 \%)$ each from peritonitis, liver lesion, cellulitis and urinary tract infection. Demographic data indicated $82.7 \%$ of the subjects were males, and $59.4 \%$ were between $20-39$ years old; 25 children less than 16 years old were reported. The prevalent risk factor was HIV infection (78.1\%). The mean annual incidence rate of cryptococcosis 
in the general population was $2.4 \times 10^{6}$ inhabitants, but in AIDS patients the rate rose to one in $3 \times 10^{3}$. The most frequent clinical features were headache $(85.2 \%)$, nausea and vomiting $(59.1 \%)$, fever $(59.0 \%)$, mental changes (46.2\%), meningeal signs $(33.4 \%)$, cough $(23.6 \%)$ and visual alterations or loss of vision $(20.9 \%)$. Laboratory data showed that direct examination of cerebrospinal fluid (CSF) was positive in $92.8 \%$ cases and Cryptococcus was recovered in $90.3 \%$ of the cases. Cryptococcal antigen reactivity was $98.9 \%$ in CSF and $93.7 \%$ in serum samples. From 788 isolates submitted, $95.9 \%$ were $C$. neoformans var. grubii serotype A, $0.3 \%$ var. neoformans serotype D, 3.3\% C. gattii serotype B and $0.5 \%$ C. gattii serotype C. The majority of patients were treated initially with amphotericin $B$.

Conclusion. Cryptococcosis incidence has increased dramatically in Colombia with the AIDS pandemic and it can be considered as a sentinel marker for HIV infection.

Key words: Cryptococcosis, Cryptococcus neoformans, AIDS, morbidity, mortality, Colombia.

La criptococosis es una infección que se adquiere por la inhalación de los propágulos infectantes de las dos especies y dos variedades del complejo de especie Cryptococcus neoformans, a saber, C. neoformans var. grubii, serotipo A, C. neoformans var. neoformans, serotipo $D$, el híbrido AD y C. gattii, serotipos B y C. La historia natural de la criptococosis no está todavía completamente elucidada, pero numerosos estudios publicados señalan que existen diferencias entre las especies y variedades de acuerdo con el tipo de hospedero y el daño orgánico resultante. En la mayoría de los pacientes con afección predisponente prevalece $C$. neoformans var. grubii mientras que $C$. gattii afecta principalmente a hospederos inmunocompetentes (1-6).

Se considera que el $10 \%$, y en algunas regiones hasta el 30\%, de los pacientes infectados con el virus de la inmunodeficiencia humana $(\mathrm{VIH})$ que no reciben terapia antirretroviral de alta efectividad (HAART, por su sigla en inglés de Highly Active AntiRetroviral Therapy) pueden desarrollar criptococosis, documentándose ésta como la enfermedad oportunista que define el sida en un número importante de casos (7-9). El compromiso meníngeo, así como el sistémico, es de especial gravedad y, por lo tanto, la morbilidad y la mortalidad son altas $(10,11)$. El uso generalizado de la HAART en los países industrializados ha producido una disminución significativa en la incidencia de criptococosis (12-15).

\footnotetext{
Correspondencia:

Jairo Lizarazo, Calle 13 No. 1E-44, consultorio 404B, Cúcuta, Norte de Santander. Teléfono: 5722635, fax: 5835161 jflizar1@telecom.com.co

Recibido: 06/10/06; aceptado: 20/12/06
}

La criptococosis no es enfermedad de notificación obligatoria en Colombia, lo que explica porqué existe tan poca información sobre su incidencia en los grupos de mayor riesgo. Sin embargo, en la literatura nacional se encuentran una serie de artículos que revelan la importancia de la criptococosis en nuestro medio (16-22).

Con el fin de identificar las características demográficas y la procedencia de la población colombiana afectada por la criptococosis, y conocer los factores de riesgo, la extensión de la diseminación al momento del diagnóstico, los métodos de laboratorio empleados para establecer el diagnóstico, la especie de Cryptococcus agente causal y el tratamiento inicial de los pacientes infectados con C. neoformans, en 1997 se diseñó una encuesta, la cual ha sido diligenciada por los profesionales que hoy constituyen el Grupo Colombiano de Estudio de la Criptococosis.

El objetivo de este trabajo es el de presentar el análisis epidemiológico y clínico de los datos de los 931 pacientes en los que se ha diligenciado la encuesta, la cual destaca la importancia de la criptococosis en nuestro medio. Los resultados de la encuesta han sido presentados en reuniones nacionales e internacionales y los datos a diciembre de 1999 fueron publicados en el Informe Quincenal Epidemiológico Nacional (IQEN) (23).

\section{Materiales y métodos \\ Diseño del estudio}

Este es un estudio observacional descriptivo en el que se recogió la información clínica y epidemiológica de los casos de criptococosis diagnosticados en el país de 1997 a 2005. La 
información fue obtenida a través de una encuesta diseñada de acuerdo con las pautas establecidas por la Confederación Europea de Micología Médica, con la autorización correspondiente (24), y diligenciada por clínicos y bacteriólogos de hospitales públicos, universitarios y privados, y de laboratorios de salud pública del país.

En la encuesta se consignaron datos demográficos de los pacientes, sexo, año y departamento de nacimiento, departamento de residencia, factores de riesgo (sida, terapia con corticoesteroides, enfermedad autoinmune, trasplante, tumor sólido, malignidad hematológica, diabetes mellitus, falla renal y cirrosis), fecha del diagnóstico clínico, signos y síntomas (fiebre, cefalea, náuseas o vómito, confusión, cambios mentales, tos, visión anormal o pérdida de la visión, focalización neurológica, signos meníngeos, hidrocefalia o hipertensión intracraneana, convulsiones); hallazgos radiológicos del tórax, tomografía computarizada (TAC) y resonancia magnética (RM). Los datos del laboratorio incluían origen de la muestra positiva, resultado del examen directo, del cultivo y de la antigenemia y el recuento de células CD4+ por ml. También se solicitó información sobre el tratamiento antirretroviral y el tratamiento inicial para la criptococosis (25).

Las encuestas y los aislamientos del hongo fueron remitidos a los dos centros coordinadores del estudio, a saber, el Instituto Nacional de Salud y la Corporación para Investigaciones Biológicas de Medellín. Con estos datos se creó una base en Epiinfo 6.0, programa que se empleó para el análisis (26).

En la literatura mundial se ha reportado que la criptococosis en niños es un evento poco común (27), por ese motivo decidimos realizar un análisis adicional de la población infantil con criptococosis. De igual manera, se presentan las principales características de los casos ocasionados por C. gattii.

\section{Definición de caso}

El caso se definió con base en la presencia de hallazgos clínicos compatibles con criptococosis, además de la presencia de uno o varios de los siguientes resultados de laboratorio: aislamiento de C. neoformans de un lugar normalmente estéril, o de esputos, lavados broncoalveolares, de una lesión de la piel, o la visualización del hongo en el examen directo con tinta china del LCR, o de una lesión cutánea, o de un examen histopatológico, así como un título de antígeno capsular $\geq 8$ en suero o LCR.

Se consideraron como recaídas los episodios clínicos que se presentaron seis o más meses después del diagnóstico inicial (28).

El análisis de la información de la encuesta se realizó como casos de sida cuando la infección con el VIH estaba consignada en la encuesta y no sida en los casos negativos para la infección con el VIH, o en los que esta información no había sido consignada.

\section{Análisis epidemiológico}

La incidencia anual de la criptococosis se calculó dividiendo el número de casos registrados por la proyección de la población para el año de acuerdo con el DANE (29). La incidencia anual de los casos de criptococosis asociada con sida se calculó con base en el número de pacientes con criptococosis como numerador y el número de pacientes que vivían con el VIH en el denominador. Los datos de los casos de VIH fueron obtenidos del Instituto Nacional de Salud (30). Para las incidencias promedio anuales se utilizaron como denominadores las respectivas poblaciones para el 2001, año intermedio del tiempo de la vigilancia epidemiológica.

Para el análisis, el país fue dividido en siete regiones, a saber: 1. Bogotá D.C., Cundinamarca y Boyacá; 2. Antioquia y Eje Cafetero (Caldas, Risaralda y Quindío); 3. Costa Pacífica (Chocó, Valle, Cauca y Nariño); 4. Santanderes (Santander y Norte de Santander); 5. Tolima y Huila; 6 . Costa Caribe y Territorio Insular (Cesar, La Guajira, Magdalena, Atlántico, Bolívar, Sucre, Córdoba y San Andrés y Providencia) y 7 . Amazonia-Orinoquia (Amazonas, Vaupés, Guainía, Guaviare, Putumayo, Caquetá, Meta, Casanare, Arauca y Vichada).

\section{Estudios micológicos}

Se confirmaron los aislamientos de C. neoformans con el empleo de técnicas estandarizadas en el 
laboratorio (31). La especie fue determinada mediante el cultivo en el medio de canavanina glicina - azul de bromotimol (CGB) (32). El serotipo se estableció con el empleo del estuche comercial con anticuerpos policlonales específicos (CryptoCheck, Iatron, Japón). Los aislamientos se conservaron en agua destilada estéril a temperatura ambiente y se congelaron en glicerol al $10 \%$ a $-70^{\circ} \mathrm{C}(33)$.

En 93 aislamientos, 84 de $C$. neoformans var. grubii serotipo A y 9 de $C$. gatti serotipo $\mathrm{B}$, se determinó la concentración inhibitoria mínima a la anfotericina B (AmB), al itraconazol (ITZ) y al fluconazol (FCZ) con la técnica de microdilución en caldo de acuerdo con el procedimiento e interpretación establecidos por el Comité Nacional de Estándares de Laboratorio de los Estados Unidos (NCCLS), hoy denominado Instituto para Estándares de Laboratorio Clínico (CLSI) (34). Se emplearon como cepas control $C$. neoformans ATCC 90112 y ATCC 90113.

Con algunos de los aislamientos remitidos para esta encuesta se realizaron estudios de huella molecular y determinación de pareja sexual por PCR. En un primer estudio con aislamientos iberoamericanos se incluyeron 39 cepas (35) y en el segundo 178 (36). De los 217 aislamientos, 177 $(81,6 \%)$ serotipo A se agrupaban en el patrón molecular VNI y 10 (4,6\%) en el patrón VNII; 1 (0,5\%) aislamiento serotipo $D$ en el patrón VNIV; dos $(0,9 \%)$ aislamientos serotipo $B$ en el patrón VGI; $22(10,1 \%)$ en el patrón VGII; uno en VGIII y uno en VGIV, y tres aislamientos serotipo $C$ en el patrón VGIII. En 178 aislamientos se determinó la pareja sexual; 162 aislamientos serotipo $A$ fueron pareja sexual $\alpha$, de los 15 serotipo B, el $20 \%$ fue pareja sexual a y el $80 \%$ pareja sexual $\alpha$; el único aislamiento serotipo $C$ fue pareja sexual $\alpha$.

\section{Resultados}

En el periodo de estudio se recibieron 931 encuestas de 76 centros en 25 departamentos y en Bogotá, D. C. No se registraron pacientes de siete departamentos (Cesar, La Guajira, Sucre, Putumayo, Guainía, Vaupés y Guaviare). El número de encuestas recibidas según el año fueron: 46 (4,9\%) en 1997, 79 (8,5\%) en 1998, 78 $(8,4 \%)$ en 1999, $97(10,4 \%)$ en $2000,95(10,2 \%)$ en $2001,128(13,8 \%)$ en 2002, $155(16,7 \%)$ en 2003, 168 (18,0\%) en 2004 y $85(9,1 \%)$ en 2005.

\section{Incidencia anual de la criptococosis}

Los datos obtenidos en los 9 años de análisis señalaron una incidencia anual que varió de 1,1 por millón de habitantes en 1997 hasta 3,7 en 2004. La incidencia promedio anual fue de 2,4 casos por millón de habitantes. En el cuadro 1 se señalan las incidencias anuales por regiones.

\section{Factores de riesgo}

El principal factor de riesgo fue la infección con el VIH en $727(78,1 \%)$ casos. Otros factores de riesgo reportados fueron: uso de esteroides en $38(4,1 \%)$, enfermedad autoinmune en 21 (2,3\%), tumor sólido o malignidad en $18(1,9 \%)$, transplante en $12(1,3 \%)$, diabetes mellitus en $10(1,1 \%)$, cirrosis hepática en $8(0,9 \%)$, falla renal crónica en 3 $(0,3 \%)$, otro factor en $3(0,3 \%)$, mientras que en 123 casos $(13,2 \%)$ no se determinó su existencia. En $25(2,7 \%)$ pacientes se presentó más de un factor de riesgo.

La criptococosis definió el sida en 179 (24,6\%) casos. La incidencia promedio anual de criptococosis

Cuadro 1. Criptococosis en Colombia. 1997-2005. Incidencia promedio anual por regiones.

\begin{tabular}{lccc}
\hline Regiones & Población 2001 & Casos $\mathbf{n ~ ( \% ) ~}$ & Incidencia/año10 $^{\mathbf{6}}$ \\
\hline Bogotá, Cundinamarca, Boyacá & 10.653 .051 & $308(33,1)$ & 3,2 \\
Antioquia, Eje Cafetero & 8.108 .712 & $236(25,3)$ & 3,2 \\
Costa Pacífica & 7.593 .908 & $203(21,8)$ & 3,0 \\
Santanderes & 3.365 .040 & $111(11,9)$ & 3,7 \\
Tolima, Huila & 2.240 .080 & $27(2,9)$ & 1,3 \\
Costa Caribe & 8.966 .967 & $35(3,8)$ & 0,4 \\
Amazonia, Orinoquia & 2.142 .946 & $11(1,2)$ & 0,6 \\
Colombia & 43.070 .704 & 931 & 2,4 \\
\hline
\end{tabular}


en los pacientes con sida fue de 3,0 casos por mil. La incidencia promedio anual por regiones en pacientes con sida se consigna en el cuadro 2. En la figura 1 se señalan por regiones las incidencias de criptococosis, tanto en la población general como en los pacientes infectados por el VIH.

El número de casos de criptococosis observados en los 9 años de la encuesta en la población

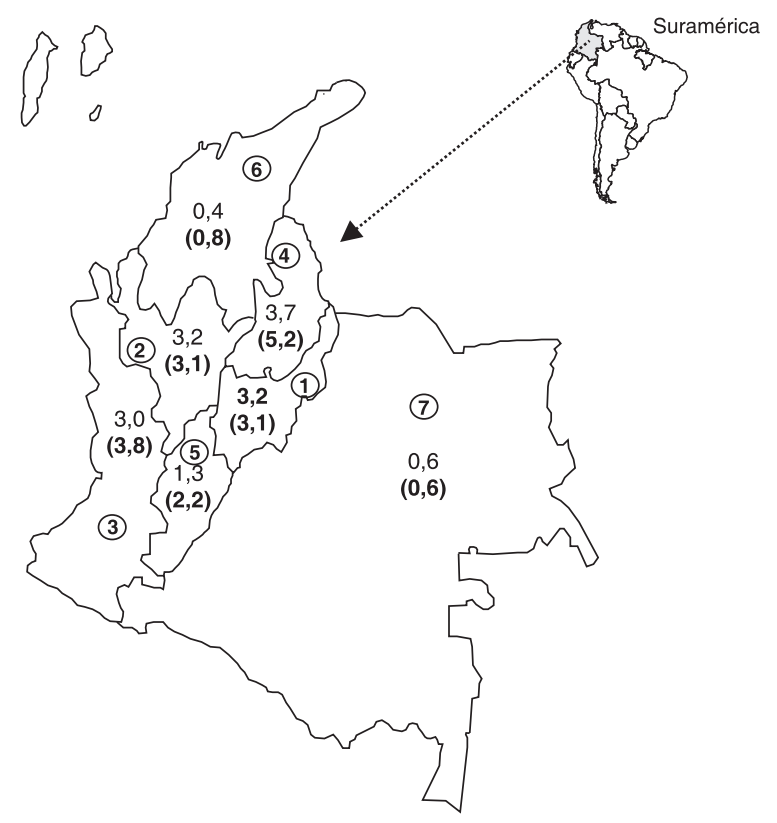

Figura 1. Incidencia de criptococosis en Colombia por regiones. Incidencia promedio anual $\times 10^{6}$ e incidencia promedio anual en pacientes con sida indicada en paréntesis x $10^{3}$. Regiones indicadas en círculos: 1. Bogotá, Cundinamarca, Boyacá; 2. Antioquia, Eje Cafetero; 3. Costa Pacífica; 4. Santanderes; 5. Tolima, Huila; 6. Costa Caribe; 7. Amazonia, Orinoquia. general y en los pacientes con sida y sin sida se presentan en la figura 2.

\section{Datos demográficos}

La distribución de los pacientes por género mostró $770(82,7 \%)$ hombres y $161(17,3 \%)$ mujeres, para una proporción de 4,8:1. La proporción de género y el promedio de edad variaron según el estado de la infección con el VIH (cuadro 3).

La distribución por grupos de edad en la población general y en los pacientes según su estado de $\mathrm{VIH}$ se muestra en la figura 3.

\section{Procedencia}

La procedencia de los pacientes de acuerdo con su situación de VIH se muestra en el cuadro 4.

\section{Formas clínicas}

Se identificaron $891(95,7 \%)$ casos de neurocriptococosis, $890(99,9 \%)$ de meningitis y

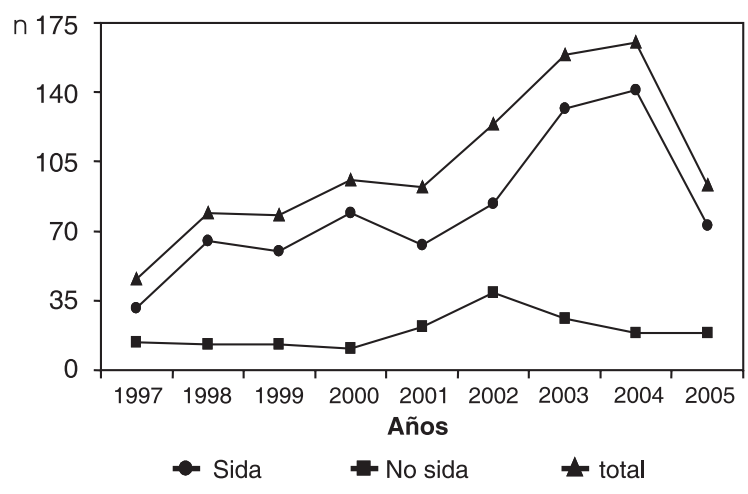

Figura 2. Situación de la criptococosis en Colombia (1997-2005). Número anual de casos según estado de la infección con el VIH.

Cuadro 2. Criptococosis en Colombia. 1997-2005. Incidencia promedio anual en pacientes VIH positivos.

\begin{tabular}{lccc}
\hline Regiones & Población VIH + & \multicolumn{2}{c}{ Casos de criptococosis } \\
\cline { 3 - 4 } & $\mathbf{2 0 0 1}$ & $\mathbf{n}(\%)$ & ${\text { Tasa } / \mathbf{1 0}^{\mathbf{3}}}$ \\
\hline Bogotá, Cundinamarca, Boyacá & 8.104 & $224(30,8)$ & 3,1 \\
Antioquia, Eje Cafetero & 6.896 & $192(26,4)$ & 3,1 \\
Costa Pacífica & 5.310 & $180(24,7)$ & 3,8 \\
Santanderes & 1.789 & $83(11,4)$ & 5,2 \\
Tolima, Huila & 903 & $18(2,5)$ & 2,2 \\
Costa Caribe & 3.480 & $26(3,6)$ & 0,8 \\
Amazonia, Orinoquia & 781 & $4(0,6)$ & 0,6 \\
Colombia & 27.263 & 727 & 3,0 \\
\hline
\end{tabular}


uno $(0,1 \%)$ de criptococoma cerebral; 27 casos $(2,9 \%)$ revelaron solamente compromiso pulmonar; cinco $(0,5 \%)$ fueron lesiones cutáneas; dos $(0,2 \%)$ ganglionares; dos $(0,2 \%)$ úlceras orofaríngeas, y un caso $(0,1 \%)$ de cada una de las siguientes formas clínicas: peritonitis, lesión hepática, celulitis de los miembros inferiores e infección de las vías urinarias.

Cuadro 3. Características de los pacientes con criptococosis diagnosticados en Colombia (1997-2005).

\begin{tabular}{lccc}
\hline Variable & Sida & No sida & Total \\
\hline Número de pacientes & 727 & 204 & 931 \\
Hombre: mujer & $7,3: 1$ & $1,8: 1$ & $4,8: 1$ \\
Edad promedio & $34,8 \pm 10,3^{*}$ & $44 \pm 19,8^{*}$ & $36,7 \pm 13,2$ \\
(años) \pm DE & & &
\end{tabular}

En 33 pacientes no se informó la edad en el momento del diagnóstico

${ }^{*} p=0,1$

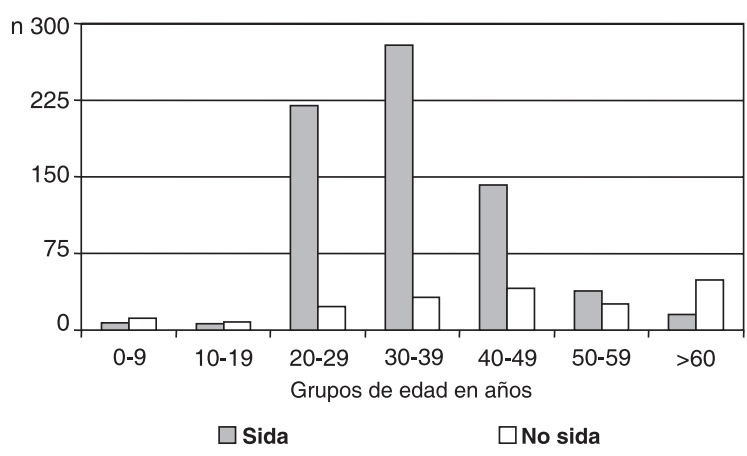

Figura 3. Encuesta nacional de criptococosis (1997-2005). Distribución por edad y estado de infección con el VIH.

\section{Síntomas y signos}

Estos datos fueron registrados en 836 (89,8\%) encuestas. En el cuadro 5 se muestran agrupados de acuerdo con el estado de la infección con el VIH.

\section{Imágenes diagnósticas}

Se reportaron los hallazgos de la radiografía del tórax, TAC de cráneo y RM de cerebro. Se realizó radiografía simple de tórax en 354 pacientes; el estudio fue normal en 215 casos $(60,7 \%)$ y anormal en 139 (39,3\%); las anormalidades descritas fueron: infiltrados pulmonares, 97; cavitaciones, 32 (tres tenían tuberculosis); nódulos, cinco; derrame pleural, tres; adenomegalias, uno, y consolidación neumónica, uno.

La neuroimágenes se reportaron en 185 encuestas: 160 TAC de cráneo y 25 RM de cerebro; 93 $(50,3 \%)$ de estos estudios fueron anormales. Las anormalidades informadas fueron: atrofia cerebral, 27 casos; infartos cerebrales, 19; granuloma cerebral, 14; hidrocefalia, 10; edema cerebral, 6; encefalitis, 3; calcificaciones, 3; dilatación de los espacios de Virchow-Robin, 1, y otras, 10.

\section{Diagnóstico micológico}

Examen directo y cultivo. El examen directo del LCR fue positivo en $784 / 845(92,8 \%)$ de los casos en los que el examen fue informado. El cultivo del LCR se realizó en 884 casos y fue positivo en 798 (90,3\%). El hongo se recuperó de otros sitios con las siguientes frecuencias: sangre, 72 casos; lavado broncoalveolar, 19; piel, 8; esputos, 3; orina, 2; líquido ascítico, 1; líquido pleural, 1, y

Cuadro 4. Criptococosis en Colombia. 1997-2005. Casos de sida y no sida por regiones.

\begin{tabular}{|c|c|c|c|c|}
\hline Regiones & Sida & $\begin{array}{c}\text { No sida } \\
\text { n (\%) }\end{array}$ & Total & $\mathbf{p}$ \\
\hline \multicolumn{5}{|l|}{ Bogotá, Cundinamarca, } \\
\hline Antioquia, Eje Cafetero & $192(81,4)$ & $44(18,6)$ & $236(25,3)$ & $<0,001$ \\
\hline Costa Pacífica & $180(88,7)$ & $23(11,3)$ & $203(21,8)$ & 0,05 \\
\hline Santanderes & $83(74,8)$ & $28(25,2)$ & $111(11,9)$ & \\
\hline Tolima, Huila & $18(66,7)$ & $9(33,3)$ & $27(2,9)$ & \\
\hline Costa Caribe & $26(74,3)$ & $9(25,7)$ & $35(3,8)$ & \\
\hline Amazonia, Orinoquia & $4(36,4)$ & $7(63,6)$ & $11(1,2)$ & \\
\hline Colombia & $727(78,1)$ & $204(21,9)$ & 931 & \\
\hline
\end{tabular}


Cuadro 5. Criptococosis en Colombia. 1997-2005. Frecuencia de síntomas y signos en los casos sida y no sida. Datos de 836 encuestas.

\begin{tabular}{lccccc}
\hline Síntomas/signos & \multicolumn{2}{c}{ Sida, N=662 } & \multicolumn{2}{c}{ No sida, $\mathbf{N = 1 7 4}$} & $\mathbf{p}$ \\
\cline { 2 - 6 } & $\mathbf{n}$ & $\mathbf{n}$ & 120 & $\%$ & $<0,001$ \\
\hline Cefalea & 588 & 88,8 & 88 & 69,0 & 0,01 \\
Nauseas/vómito & 403 & 60,9 & 82 & 47,1 & 0,03 \\
Fiebre & 408 & 61,6 & 97 & 55,7 & \\
Confusión & 287 & 43,4 & 59 & 33,9 & \\
Signos meníngeos & 219 & 33,1 & 35 & 24,1 & \\
Tos & 161 & 24,3 & 42 & 13,2 & \\
Visión anormal & 132 & 19,9 & 23 & 22,4 & \\
Convulsiones & 126 & 19,0 & 39 & 15,5 & \\
Hidrocefalia/HIC & 85 & 12,8 & 27 &
\end{tabular}

HIC: Hipertensión intracraneana

Cuadro 6. Criptococosis en Colombia 1997-2005. Positividad del diagnóstico micológico y determinación de antígeno circulante.

\begin{tabular}{lcccccc}
\hline \multirow{2}{*}{ Examen } & \multicolumn{2}{c}{ Sida } & \multicolumn{2}{c}{ No sida } & \multicolumn{2}{c}{ Total } \\
\cline { 2 - 7 } & \multicolumn{3}{c}{ n/N (\%) } \\
\hline Directo & $623 / 660$ & $(94,4)^{*}$ & $161 / 185$ & $(87,0)$ & $784 / 845$ & $(92,8)$ \\
Cultivo de LCR & $646 / 696$ & $(92,8)^{*}$ & $152 / 188$ & $(80,8)$ & $798 / 884$ & $(90,3)$ \\
Antígeno circulante en LCR & $192 / 194$ & $(99,0)$ & $78 / 79$ & $(98,7)$ & $270 / 273$ & $(98,9)$ \\
Antigenemia en suero & $69 / 72$ & $(95,8)$ & $20 / 23$ & $(87,0)$ & $89 / 95$ & $(93,7)$ \\
\hline
\end{tabular}

${ }^{*} p<0,001$

médula ósea, 1. Los resultados de estos exámenes de acuerdo con el estado de infección con el VIH se consignan en el cuadro 6 .

Determinación de antígeno circulante. Se determinó en 273 muestras de LCR con una reactividad de $98,9 \%$ (270/273) y en 95 muestras de suero con una reactividad del 93,7\% (89/95), y títulos que oscilaron entre $1: 1$ y 1:8.192 en muestras de LCR, tanto en pacientes sida, como no sida, y títulos en suero hasta 1:4.096 en pacientes sida y 1:32 en pacientes no sida. En el cuadro 6 se consignan los resultados de acuerdo con el estado de infección con el VIH.

Biopsias. Quince biopsias fueron positivas para criptococosis, 10 por cultivo y 5 por visualización. Los tejidos examinados fueron orofaringe, pulmón, hígado y ganglio linfático.

Especies, variedades y serotipos. Se remitieron un total de 788 aislamientos de los cuales 756 $(95,9 \%)$ fueron identificados como $C$. neoformans var. grubii serotipo A; 26 (3,3\%) como C. gattii serotipo B; 4 (0,5\%) como C. gattii serotipo C, y
$2(0,3 \%)$ como C. neoformans var. neoformans serotipo $D$.

En 621 pacientes con sida se identificaron los siguientes aislamientos: $C$. neoformans var grubii serotipo A en 618 (99,5\%); C. neoformans var. neoformans serotipo $D$ en $2(0,3 \%)$, y $C$. gattii serotipo $B$ en $1(0,2 \%)$.

En 167 pacientes sin sida se aislaron $C$. neoformans var. grubii serotipo A en 138 (82,6\%); C. gatti serotipo B en 25 (15,0\%), y C. gattii serotipo $C$ en $4(2,4 \%)$.

Sensibilidad antimicótica. De los 84 aislamientos de $C$. neoformans var. grubii serotipo $A$ en los que se determinó la susceptibilidad, $82(97,6 \%)$ fueron sensibles a $A m B(C I M=1,0 \mu \mathrm{g} / \mathrm{ml}), 84(100 \%)$ a ITZ (CIM=0,125 $\mu \mathrm{g} / \mathrm{ml})$ y $84(100 \%)$ a FCZ (CIM $=8,0 \mu \mathrm{g} / \mathrm{ml}$ ). De los nueve aislamientos de $C$. gattii serotipo B, $9(100 \%)$ fueron sensibles a AmB (CIM=1,0 $\mu \mathrm{g} / \mathrm{ml}), 8(88,8 \%)$ a ITZ (CIM=0,125 $\mu \mathrm{g} /$ $\mathrm{ml})$, y $8(88,8 \%)$ a FCZ $(\mathrm{CIM}=8,0 \mu \mathrm{g} / \mathrm{ml})$.

Niveles de CD4+. El recuento de células CD4+ se consignó en las encuestas de $125(17,2 \%)$ de 
los 727 pacientes infectados con el $\mathrm{VIH}$; los valores oscilaron entre 4 y 685 células $/ \mathrm{mm}^{3}$ con una mediana de 45 células $/ \mathrm{mm}^{3} ; 112$ (89,6\%) tenían cifras iguales o menores a 200 células/ $\mathrm{mm}^{3}$ y de éstos, $92(80 \%)$ presentaban valores iguales o menores a 100 células $/ \mathrm{mm}^{3}$.

Tratamiento. El dato de tratamiento estaba consignado en 737 encuestas, de las cuales, 582 $(78,9 \%)$ recibieron un solo antifúngico; 424 (72,8\%) de los casos recibieron AmB; 144 (24,7\%), FCZ y $14(2,4 \%)$, ITZ. De los $155(21 \%)$ con terapia combinada, $128(82,5 \%)$ recibieron AmB más FCZ y $27(17,4 \%)$ otras combinaciones.

Tratamiento antirretroviral. Sólo en 18 (2,5\%) de los 727 pacientes con sida se informó el suministro de terapia antirretroviral en el momento del diagnóstico de la criptococosis. No se especificó el tipo de terapia.

\section{Criptococosis en niños}

Se reportaron $25(2,7 \%)$ niños menores de 16 años, 15 (60\%) varones y 10 (40\%) niñas. La edad promedio al momento del diagnóstico fue de 7,2 $\pm 3,8$ años. El menor de los niños fue un neonato de 13 días de nacido $\mathrm{VIH}$ negativo al igual que su madre.

La frecuencia por región de origen fue la siguiente: Bogotá, D.C., Cundinamarca y Boyacá, 6; Antioquia y Eje Cafetero, 4; Costa Pacífica, 4; Santanderes, 8; Tolima y Huila 2, y Orinoquia y Amazonia, 1.

Los factores de riesgo descritos fueron los siguientes: infección por el VIH, 7 (28\%); tumor sólido, 3 (12\%); enfermedad autoinmune (LES) y uso de esteroides, 1 (4\%); no se informó factor de riesgo en 14 niños (56\%). La criptococosis definió el sida en 3 (42,9\%) de ellos. Ninguno de los pacientes infectados por el VIH estaba recibiendo terapia antirretroviral y las células CD4+ se reportaron únicamente en un paciente, con un valor de 135 células $/ \mathrm{mm}^{3}$. Se presentaron 24 (96\%) nuevos casos y una (4\%) recaída .

Al igual que en los adultos, las principales manifestaciones clínicas de los niños fueron cefalea, náuseas o vómitos, fiebre, confusión mental y signos meníngeos; no se pudieron establecer diferencias significativas debido a la heterogeneidad en los denominadores.

A 22 de los 25 niños se les realizó cultivo y el hongo se recuperó en 20 (90,9\%), 19 a partir de LCR y uno de hemocultivo; de los 20 aislamientos, $18(90 \%)$ fueron $C$. neoformans variedad grubii serotipo A y 2 (10\%), C. gattii serotipo $B$.

Los estudios imaginológicos realizados fueron radiografía de tórax en $8 / 25$ (32\%), TAC de cráneo en $7 / 25$ (28\%) y RM de cerebro en $1 / 25$ (4\%). Los hallazgos radiológicos en el tórax fueron normales en $7 / 8(87,5 \%)$ y anormales (infiltrados), en $1 / 8$ $(12,5 \%)$. En las imágenes del cerebro se determinaron infartos en tres, hidrocefalia en uno, aumento del realce meníngeo en dos, atrofia cerebral con múltiples calcificaciones en uno y aspecto normal en un niño.

El tratamiento con antifúngicos se aplicó en 21 (84\%) pacientes: sólo con AmB en $14(66,7 \%)$; con AmB más 5-fluorocitosina en tres (14,3\%); con AmB mas FCZ en tres (14,3\%), y con monoterapia con FCZ en uno (4,7\%).

\section{Criptococosis por C. gattii}

Esta especie se aisló en 30 casos: 24 (80\%) hombres y seis $(20 \%)$ mujeres. La edad promedio en el momento del diagnóstico fue de $42 \pm 17$ años con edades límites de 4 y 68 años. En este grupo hubo dos niños menores de 16 años.

Los pacientes residían en Norte de Santander, 7 (23,3\%); en Bogotá D.C., 6 (20,0\%); en Caquetá, $3(10 \%)$, y en Risaralda, Santander y Valle, 2 $(6,7 \%)$ en cada uno. Los siguientes departamentos presentaron un $(3,3 \%)$ caso: Antioquia, Arauca, Bolívar, Boyacá, Caldas, Cauca, Córdoba y Meta.

No se conoció el factor de riesgo en 28 (93,3\%) casos. Sólo un $(3,3 \%)$ paciente tenía infección por el VIH y le definió sida; en otro $(3,3 \%)$ caso se informó diabetes mellitus.

Las formas clínicas de presentación fueron meningitis en $28(93,3 \%)$ y casos pulmonares en dos $(6,7 \%)$. Los síntomas y signos se determinaron en 29 casos y fueron los siguientes en orden de frecuencia: cefalea en 22 (75,9\%); náusea y vómitos en 18 (62,1\%); confusión mental en 16 (55,2\%); pérdida de visión o visión anormal en 15 
(51,7\%); fiebre en 11 (37,9\%); signos meníngeos en $10(34,5 \%)$; hidrocefalia o hipertensión intracraneal en $9(31,0 \%)$; convulsiones en 6 $(20,7 \%)$; focalización neurológica en $6(20,7 \%)$, y tos en $3(10,3 \%)$.

C. gattii fue recuperado del LCR en 28 (93,3\%) casos y del pulmón en dos (6,7\%). En uno de los casos de meningitis también se obtuvo el hongo a partir de un hemocultivo. Veintiséis $(86,7 \%)$ aislamientos fueron serotipo B y cuatro $(13,3 \%)$, serotipo C. El examen directo del LCR fue positivo en $27 / 28(96,4 \%)$ casos. La determinación del antígeno capsular fue reactiva en $5 / 6(83,3 \%)$ en el suero y en $10 / 10(100 \%)$ en LCR.

La radiografía de tórax fue normal en 10/13 $(76,9 \%)$ y mostró lesiones pulmonares en $3 / 13$ $(23,1 \%)$. Las imágenes diagnósticas del cerebro demostraron diferentes lesiones en 10/13 (76,9\%).

Se conoció del tratamiento inicial en $26(86,7 \%)$ casos; 14 (46,7\%) recibieron únicamente AmB, 6 (20\%) fueron tratados con AmB más FCZ, $3(10 \%)$ sólo con FCZ, 1 (3,3\%) con AmB más FCZ más ITZ, 1 (3,3\%) con AmB más 5-fluorocitosina y 1 (3,3\%) sólo con ITZ.

\section{Discusión}

En 1978, Kaufman del CDC de Atlanta llamaba la atención sobre la criptococosis comparándola con el despertar de un gigante por la frecuencia con que comenzaba a diagnosticarse en pacientes inmunológicamente comprometidos (37); 28 años después, Levitz y Boekhout, en su editorial de FEMS Yeast dedicado a la criptococosis confirmaron lo vaticinado por Kaufman (38). Los datos presentados en este artículo señalan igualmente que en nuestro país, la criptococosis ocasiona gran morbilidad y mortalidad.

Esta encuesta constituye la primera aproximación nacional al conocimiento epidemiológico y clínico de la criptococosis. No fue posible determinar el grado de precisión de la encuesta con respecto al número real de casos de criptococosis ocurridos en Colombia durante el período de la vigilancia, pues no existen otras bases de datos de casos de criptococosis que permitan dicha comparación. Utilizando el método de captura-recaptura de Dromer et al. (7), se ha estimado que un tipo de encuesta pasiva como la nuestra, en la que participan en forma voluntaria clínicos y bacteriólogos, tiene una precisión cercana al $50 \%$. Cuando existe un registro nacional obligatorio de casos de sida y criptococosis, la combinación con los datos de la encuesta puede alcanzar una representación de más de $80 \%$ (7), lo cual no es nuestro caso. Es importante llamar la atención sobre la necesidad de realizar la notificación de éste y otros eventos de importancia al sistema de vigilancia en salud pública, SIVIGILA (39), para poder validar este tipo de eventos.

En los primeros ocho años de la encuesta (19972004) se observó una tendencia al aumento progresivo de casos nuevos, hecho que se presentó paralelamente con el aumento de la incidencia de los casos asociados con sida. En el año 2005, último de este análisis, se notó una disminución tanto en el número total de casos como en el de los asociados con sida, sin mayor cambio en el número de casos no sida (figura 1). Esto tal vez se deba a la mayor cobertura de la terapia antirretroviral de alta eficacia HAART y, en menor grado, a un mayor uso de los azoles. La persistencia de valores similares en los casos de criptococosis en pacientes sin sida favorecería esta hipótesis. En países industrializados, la HAART ha demostrado ser la medida más eficaz para disminuir el número de casos de infecciones oportunistas, entre ellas la criptococosis, en pacientes que conviven con el $\mathrm{VIH}$; en Francia, por ejemplo, se ha calculado que la incidencia de criptococosis ha disminuido en un $46 \%$ después de la introducción de la HAART (12). Se observa con sorpresa que Brasil, un país en vías de desarrollo con un programa nacional exitoso para el tratamiento del $\mathrm{VIH} /$ sida y en donde el acceso a la HAART es universal, no se ha dado este cambio y las infecciones del sistema nervioso siguen presentándose con la misma frecuencia que antes de la introducción de la HAART (40).

La incidencia de la criptococosis en Colombia es baja comparada con la de otros países. En 1992, en el área metropolitana de Atlanta, Estados Unidos, se reportaron incidencias anuales de 66 casos por mil personas que vivían con el VIH; estas incidencias bajaron a siete en el año 2000 (41). Sin embargo, esta última cifra es superior al 
promedio anual de 3 casos por mil personas que conviven con el VIH en Colombia. Ni siquiera la región de los Santanderes, con una incidencia anual promedio de 5,2 casos, superó dicho valor.

En Europa, las incidencias iniciales fueron menores y también cayeron con el advenimiento de la HAART. Una encuesta realizada en Italia entre 1997 y 1999 después de la introducción de la HAART, mostró una incidencia anual de 8,5 casos por mil personas que convivían con el VIH (42).

Australia y Nueva Zelanda han reportado cifras similares a las nuestras; un estudio prospectivo (43) realizado entre 1994 y 1997 (la HAART fue introducida en 1996 en ese país) señaló una incidencia promedio anual de 6,6 casos por millón de habitantes para Australia y de 2,2 en Nueva Zelanda; es necesario resaltar que sólo el $42,9 \%$ de estos pacientes tenía sida, hecho que contrasta con las cifras de casi $80 \%$ reportadas en Europa, en Estados Unidos $(7,41)$ y en esta encuesta colombiana. En el África Subsahariana la situación es dramática; la incidencia de la infección por VIH sigue en aumento y de igual forma la de la criptococosis. Por ejemplo en Uganda, una cohorte reciente mostró una incidencia anual de 40 casos por mil personas con VIH y la criptococosis fue la causa de muerte en el $17 \%$ de esta población (44).

En Colombia, las regiones con incidencias más altas fueron los Santanderes, la región Central, incluida Bogotá, y la región de Antioquia y el Eje Cafetero. Creemos que estas cifras revelan solamente una mayor notificación de casos, y que las incidencias bajas de otras regiones, como la la Costa Atlántica, se deben a una baja notificación y a dificultades en el diagnóstico micológico. Las regiones señaladas al principio han sido las que más reportan casos de infección por VIH en Colombia (30).

La infección por Cryptococcus en Colombia ocurre principalmente en hombres jóvenes, lo que refleja la epidemiología de la infección por el VIH. En la población general el predominio del género masculino es marcado, relación que aumenta en los pacientes VIH positivos; no obstante, en los pacientes sin sida se mantiene el predominio de los hombres, aunque en una proporción menor.
Este último hecho ya se reconocía desde antes de la aparición del VIH. El promedio de edad es mayor en los pacientes sin sida, en los que se da una distribución por edades más amplia de la enfermedad. La información de otros países coincide con estas observaciones $(7,9,45)$.

En esta encuesta se identificaron pacientes en las edades extremas de la vida, entre ellos 25 niños, grupo poblacional en el que pocas veces se reporta criptococosis, incluso si conviven con sida. En un estudio retrospectivo de ocho años realizado en Estados Unidos en niños $\mathrm{VIH}$ positivos, sólo el $0,85 \%$ desarrolló criptococosis (27); un estudio más reciente encontró $10(0,67 \%)$ niños menores de 16 años (4 de ellos con sida) entre 1.491 casos de criptococosis (41). En Australia y Nueva Zelanda sólo encontraron dos niños $(0,56 \%)$ menores de nueve años en una muestra de 355 casos (43). Nuestra cifra de prevalencia del $2,7 \%$ de criptococosis en niños menores de 16 años contrasta con las del $24 \%$ y $33 \%$ registradas en Brasil $(46,47)$. En el primero de estos informes se trató de niños menores de 9 años, casi la mitad de ellos afectado por $C$. gattii (46); en el segundo informe, tal porcentaje correspondía a menores de 16 años en una serie en donde la mayoría de los pacientes no tenían factor predisponente (47). Se desconoce la razón de la baja incidencia de criptococosis en niños en la mayoría de los países. Se ha demostrado que no se debe a falta de exposición, ya que en un estudio realizado en el distrito del Bronx de Nueva York, la mayoría de los niños mayores de dos años tuvieron anticuerpos contra el polisacárido de C. neoformans (48). Es llamativa la notificación de un neonato sin sida, hecho excepcional en la epidemiología de esta enfermedad $(49,50)$.

Como se ha informado en otras regiones del mundo, el principal factor de riesgo para la criptococosis en Colombia fue la infección por el VIH $(7,41,43)$. En las regiones de Antioquia, Eje Cafetero y Costa Pacífica la asociación de criptococosis y sida fue significativamente mayor que en el resto del país (cuadro 4).

En el grupo de los pacientes sin sida de nuestra encuesta aparecen otros factores de riesgo conocidos como el uso de esteroides, las 
malignidades y los transplantes, entre otros (5154). Al igual que en otros países, $5,2 \%$ en Francia (7) y $18 \%$ en Estados Unidos (41), existe un porcentaje significativo de pacientes, $13,2 \%$ en nuestro país, en el que no se determinó un factor de riesgo. La criptococosis fue la infección oportunista que definió sida en un $24,6 \%$. En la literatura mundial se han señalado cifras de 16,5\% para Francia (7), 19\% para Tailandia (55), $39 \%$ para Estados Unidos (41) y $91 \%$ para Uganda (44). Como se sabe, los pacientes con sida desarrollan la criptococosis cuando tienen una profunda inmunosupresión $(7,43)$. Los datos de los niveles de CD4 de esta encuesta confirman este hecho.

Los síntomas y signos más frecuentes señalan fundamentalmente el compromiso del sistema nervioso central de los pacientes. La cefalea, el síntoma más frecuente, y su presencia en pacientes con $\mathrm{VIH}$, nos debe alertar sobre la posibilidad de meningitis por Cryptococcus. Un estudio hecho en Brasil mostró que en $40 \%$ de los pacientes hospitalizados con $\mathrm{VIH}$, la cefalea era producto de la criptococosis del sistema nervioso central (56). Las náuseas y el vómito, la fiebre y la confusión mental siguieron en frecuencia en esta encuesta, de forma similar a lo descrito en otros estudios $(2,10,55,57)$. Tal y como se señaló previamente, los signos meníngeos son de poca ayuda clínica en el diagnóstico de la criptococosis meníngea, ya que sólo un tercio de los pacientes los presentan (44). Es significativo que únicamente $15 \%$ de nuestros pacientes tuviera hipertensión intracraneana asociada o no con hidrocefalia, puesto que se ha comunicado que hasta $60 \%$ de los pacientes con sida y criptococosis presentan hipertensión intracraneal sin hidrocefalia (58). Una explicación posible es que no se lleve a cabo sistemáticamente la medición de la presión de apertura durante la punción lumbar diagnóstica. La omisión de esta práctica tiene gravísimas consecuencias en el manejo de tales pacientes ya que la hipertensión intracraneana no tratada es una de las causas más importantes de fracaso terapéutico y de muerte $(59,60)$. En este estudio los pacientes con sida presentaron una frecuencia significativamente mayor de cefalea y fiebre (cuadro 5), hecho que se puede atribuir a la gran carga micótica que a menudo se observa en ellos (7).

En los estudios de imágenes diagnósticas, la radiografía del tórax fue anormal en un tercio de los pacientes debido al compromiso del pulmón por el hongo o por la infección concomitante por otro patógeno como Mycobacterium tuberculosis. En general, en los pacientes con sida se han descrito lesiones pulmonares más diseminadas que en los inmunocompetentes, con un comportamiento clínico más agresivo y una recuperación más lenta (61).

Las imágenes del cerebro obtenidas por TAC o RM sirven para descartar otras patologías o para detectar criptococomas o complicaciones como la hidrocefalia y los infartos. Frecuentemente son normales o sólo evidencian la atrofia encefálica propia de los pacientes con sida (62).

Como lo demuestra esta encuesta, el diagnóstico de la criptococosis es sencillo. La altísima sensibilidad del examen directo con tinta china en el LCR, especialmente en pacientes con VIH, hace obligatorio este examen, el cual es rápido y de bajo costo (63). De igual suerte, la determinación del antígeno capsular en LCR y suero tiene un alto rendimiento (63); en pacientes VIH positivos con cefalea, la determinación rápida del antígeno capsular en suero logra el diagnóstico, lo que tiene especial validez si se tienen en cuenta las dificultades para la obtención de imágenes diagnósticas del cerebro y la realización de la punción lumbar en nuestro medio. Los pacientes con sida tienen dificultad para eliminar el antígeno capsular y frecuentemente tienen títulos muy altos, no sólo en el LCR sino también en el suero (64). En nuestra encuesta, los resultados positivos para Cryptococcus en el examen directo y el cultivo del LCR en los pacientes con sida fueron significativamente mayores que los de pacientes sin sida, hecho que se explica porque usualmente la cantidad de levaduras presentes en el LCR de los primeros es mayor (7).

En cuanto a la serotipificación, se remitieron un número importante de aislamientos. Casi todos los aislamientos de los pacientes con sida correspondieron a $C$. neoformans var grubii, situación 
observada también en otras latitudes $(7,41)$. En los pacientes no sida esta variedad también fue mayoritaria; sin embargo, existió un número significativo de casos ocasionados por $C$. gattii, lo que se explica por nuestra condición de país tropical $(63,65)$. Previamente se había determinado que en algunas regiones, como en el departamento de Norte de Santander, esta especie era mayoritaria (77\%) en los pacientes no sida (20). En esta encuesta, este departamento siguió siendo el que remitió mayor número de encuestas de criptococosis por $C$. gattii.

Los pacientes afectados por $C$. gattii pocas veces tienen sida (65), lo que se corrobora en este estudio, pues solamente uno de los 30 casos presentaba infección por VIH. Las manifestaciones clínicas fueron similares a las observadas en los casos de $C$. neoformans var. grubii, y no hubo una mayor cantidad de criptococomas pulmonares como se ha descrito en otros estudios (43). En nuestra encuesta, sólo hubo un caso de criptococoma cerebral, pero no se obtuvo aislamiento.

Según los resultados de la encuesta, $C$. neoformans var. neoformans serotipo $D$ rara vez produjo enfermedad, contrario a lo descrito en Europa (7). Algunos estudios recientes de nuestro grupo demostraron que el patrón molecular VNI predominó entre los aislamientos del serotipo $A$ $(37,38)$, lo que concuerda con lo reportado en la literatura $(66,67)$ en torno a que este genotipo es la principal causa de criptococosis en pacientes inmunosuprimidos. Por otra parte, el surgimiento del predominio del patrón molecular VGII entre los aislamientos de $C$. gattii serotipo $B(37,38)$ es similar a lo encontrado en la Isla de Vancouver, Canadá, donde se reportó un brote de criptococosis ocasionado por este genotipo (68).

La AmB fue la droga más ampliamente usada en los pacientes de la encuesta. Un 45\% recibió también FCZ, en la mayoría de los casos como tratamiento secuencial. En Colombia no se dispone de la 5-fluorocitosina, lo que explica su bajísima utilización. El tratamiento aceptado en la actualidad para los pacientes con sida es el uso secuencial de AmB con o sin 5-fluorocitosina por dos semanas, seguido de fluconazol durante ocho semanas; la profilaxis secundaria con FCZ se mantiene mientras persista la inmunosupresión $(69,70)$. El tratamiento secuencial se ha extrapolado a los pacientes sin sida. Esta encuesta no evaluó los resultados del tratamiento. No obstante, la determinación de la supervivencia a seis meses de un subgrupo de 91 pacientes en la encuesta mostró que en los pacientes con sida ésta fue del $43 \%$ y para los $\mathrm{VIH}$ negativos del $47 \%$; la mayoría de los decesos ocurrieron en las primeras 4 semanas (71). Estas cifras indican un diagnóstico tardío y, probablemente, tratamientos insuficientes, incluídas la detección tardía y la falta de una terapia agresiva de la hipertensión intracraneana.

Los resultados de esta encuesta son una aproximación importante al conocimiento de la criptococosis en Colombia y constituyen, además, un indicador aceptable de los resultados del tratamiento de la infección del $\mathrm{VIH} /$ sida en nuestro país.

\section{Conflicto de intereses}

Los autores declaramos que no tenemos conflictos de intereses.

\section{Fuentes de financiación}

Este trabajo fue financiado con recursos de Instituto Nacional de Salud, la Corporación para Investigaciones Biológicas de Medellín y el Hospital Universitario Erasmo Meoz de Cúcuta.

\section{Agradecimientos}

Los autores desean expresar sus agradecimientos a los revisores anónimos cuyos comentarios permitieron aclarar muchos de los datos presentados y así mejorar el manuscrito.

\section{${ }^{4}$ Grupo Colombiano para el Estudio de la Criptococosis}

Bogotá: Sandra Huérfano, Microbiología, Gerzaín Rodríguez, Patología, Instituto Nacional de Salud. Nidia Torres, Claudia Linares, Carlos Álvarez, Erick Sánchez, Hospital San Ignacio.

Clímaco Ernesto Ojeda, Clínica San Pedro Claver, Instituto de Seguro Social.

Sandra Núñez, LSP de Bogotá.

Mónica Gutiérrez, Fundación Santa Fe de Bogotá. Henry Mendoza, José Fernando Muñoz, Hospital San José. 
Stella Mariño, Hospital de La Victoria.

Pilar Rivas, Jorge Cortés, Instituto Nacional de Cancerología.

Mauricio Zuñiga, Clínica El Bosque.

Felipe Arboleda, Clínica Shaio.

Pablo Lorenzana, Clínica Marly.

Elsy Ospina, Laboratorio Andrade Narváez. María Isabel Medina, Hospital Simón Bolívar

Bolívar: Mario Mendoza, Hospital Universitario de Cartagena.

Antioquia: Myrtha Arango, Ángela María Tobón, Corporación para Investigaciones Biológicas, Medellín.

Sergio Jaramillo, Alejandro Vélez, Carlos Andrés Agudelo, Hospital Pablo Tobón Uribe.

Ana Lucía Correa, Clínica León XIII, Hospital Pablo Tobón Uribe.

Santiago Estrada, Congregación Mariana.

Marcela Gaviria, Clínica UPB.

Jaime Robledo, Clínica CES.

Jaime Sanpedro, Hospital La María.

Roberto Panesso, Clínica Las Américas.

Liliana Franco, Hospital San Vicente de Paul.

Antonio de Castro, CIMA, Medellín.

Santander: Martha Jácome, Hospital Universitario de Santander.

Martha Rincón, Bucaramanga.

Norte de Santander: María Claudia Rodríguez, Yenny Peña, Hospital Universitario Erasmo Meoz, Cúcuta.

Valle: Jaime Galindo, Corporación de Lucha contra el Sida, Cali.

Nancy Villamarín, Hospital Universitario del Valle, Cali.

Mónica Recalde, Claudia Rocío Castañeda, Fundación Clínica Valle de Lili.

Risaralda: Mercedes Cano Henao, Comfamiliar.

Caldas: Martha Cecilia Kogson, Hospital Santa Sofía.

Magdalena: Hernando Estrada.

Córdoba: Máximo Mercado.

\section{Referencias}

1. Kwon-Chung KJ, Varma A. Do major species concepts support one, two or more species within Cryptococcus neoformans? FEMS Yeast Res 2006;6:574-87.

2. Speed B, Dunt D. Clinical and host differences between infections with the two varieties of Cryptococcus neoformans. Clin Infect Dis 1995;21:28-34.
3. Mitchell DH, Sorrell TC, Allworth AM, Heath $\mathrm{CH}$, McGregor AR, Papanaoum K et al. Cryptococcal disease of the CNS in immunocompetent hosts: influence of cryptococcal variety on clinical manifestations and outcome. Clin Infect Dis 1995;20:611-6.

4. Rozenbaum R, Goncalvez AJR, Wanke B, Caiuby MJ, Clemente H, Lazera MS et al. Cryptococcus neoformans varieties as agents of cryptococcosis in Brazil. Mycopathology 1992;119:133-6.

5. Lalloo D, Fisher D, Naraqi S, Laurenson I, Temu P, Sinha A et al. Cryptococcal meningitis ( $C$. neoformans var. gattii) leading to blindness in previously healthy Melanesian adults in Papua New Guinea. Q J Med 1994;87:343-9.

6. Rex JH, Larsen RA, Dismukes WE, Cloud GA, Bennett JE. Catastrophic visual loss due to Cryptococcus neoformans meningitis. Medicine (Baltimore) 1993;72:207-24.

7. Dromer F, Mathoulin S, Dupont B, Laporte A. Epidemiology of cryptococcosis in France: a 9-year survey (1985-1993). French Cryptococcosis Study Group. Clin Infect Dis 1996;23:82-90.

8. Currie BP, Casadevall A. Estimation of the prevalence of cryptococcal infection among patients infected with the human immunodeficiency virus in New York City. Clin Infect Dis1994;19:1029-33.

9. Hakim JG, Gangaidzo IT, Heyderman RS, Mielke J, Mushangi E, Taziwa A et al. Impact of HIV infection on meningitis in Harare, Zimbabwe: a prospective study of 406 predominantly adult patients. AIDS 2000;14: 1401-7.

10. Moosa MY, Coovadia YM. Cryptococcal meningitis in Durban, South Africa: a comparison of clinical features, laboratory findings, and outcome for human immunodeficiency virus (HIV)-positive and HIVnegative patients. Clin Infect Dis 1997;24:131-4.

11. Heyderman RS, Gangaidzo IT, Hakim JG, Mielke J, Taziwa A, Musvaire $\mathbf{P}$ et al. Cryptococcal meningitis in human immunodeficiency virus-infected patients in Harare, Zimbabwe. Clin Infect Dis 1998;26:284-9.

12. Dromer F, Mathoulin-Pelissier S, Fontanet A, Ronin $\mathrm{O}$, Dupont B, Lortholary $\mathrm{O}$ et al. Epidemiology of HIV-associated cryptococcosis in France (1985-2001): comparison of the pre- and post- HAART eras. AIDS 2004;18:555-62.

13. Maschke M, Kastrup O, Esser S, Ross B, Hengge U, Hufnagel A. Incidence and prevalence of neurological disorders associated with HIV since the introduction of highly active antiretroviral therapy (HAART). J Neurol Neurosurg Psychiatry 2000;69:376-80.

14. d'Arminio Monforte A, Duca PG, Vago L, Grassi MP, Moroni M. Decreasing incidence of CNS AIDSdefining events associated with antiretroviral therapy. Neurology 2000;54:1856-9. 
15. Kaplan JE, Hanson D, Dworkin MS, Frederick T, Bertolli $\mathbf{J}$, Lindegren ML et al. Epidemiology of human immuno-deficiency virus associated opportunistic infections in the United States in the era of highly active antiretroviral therapy. Clin Infect Dis 2000;30 (Suppl. 1):5-14.

16. Uribe P, Restrepo A, Díaz G. Estudio prospectivo clínico y microbiológico de las meningitis subagudas y crónicas. Antioquia Médica 1973;23:153-64.

17. Greer DL, de Polanía LA. Criptococosis en Colombia: Resumen de la literatura y presentación de doce casos en el Valle del Cauca. Acta Med Valle 1977; 8:160-6.

18. Vergara I, Saavedra M, Saravia J, González G, Lorenzana P, Acosta C et al. Criptococosis del sistema nervioso central. Estudio de 32 casos 19751991. Acta Med Colomb 1993;18:199-210.

19. Saavedra M, Vergara I. Alteraciones neuro-oftalmológicas en la criptococosis del sistema nervioso central. Acta Neurol Colomb 1992;8:134-41.

20. Lizarazo J, Rodríguez MC, Ordóñez N, Vargas JJ, Castañeda N. Meningitis por Cryptococcus en el Hospital Erasmo Meoz de Cúcuta. Acta Neurol Colomb 1995;11:259-67.

21. Ordóñez N, Torrado E, Castañeda E. Criptococosis meníngea de 1990 a 1995. Hallazgos del laboratorio. Biomédica 1996;16:93-7.

22. Lizarazo J, Mendoza M, Palacios D, Vallejo A, Bustamante A, Ojeda E et al. Criptococosis ocasionada por Cryptococcus neoformans variedad gattii. Acta Med Colomb 2000;25:171-8.

23. Castañeda E, Torrado E, Arango M, de Bedout C, Tobón AM, Restrepo A et al. Criptococosis en Colombia: estudio interinstitucional. Inf Quinc Epidemiol Nac 2000;5:115-9.

24. Viviani MA. Epidemiological working groups of ECMM. Mycology Newsletter 1997;0:4-5.

25. INS. Manual para el envío de aislamientos y muestras al Grupo de Microbiología. (Noviembre 30 de 2006). Disponible en: www.ins.gov.co/pdf/rnl/Micro_manual_ de_vigilancia.pdf.

26. Dean AG, Dean JA, Columbier D, Burton AH, Brendel KA, Smith DC et al. Epi Info version 6.0: a word processing, data-base and statistics program for epidemiology on microcomputers. Atlanta, Gerogia: Centers for Disease Control and Prevention; 1994.

27. Gonzalez CE, Shetty D, Lewis LL, Mueller BU, Pizzo PA, Walsh TJ. Cryptococcosis in human immunodeficiency virus-infected children. Pediatr Infect Dis J 1996;15:796-800.

28. Viviani MA. Epidemiological working groups of ECMM Mycology Newsletter 1998;2:6-7.
29. Dane. Proyecciones de población. (Agosto 04 de 2006). Disponible en: http://200.21.49.233/website/mgn2/ main6.asp.

30. Góngora LR. Informe evento VIH/SIDA Semana epidemiológica 1 a 14, primer semestre julio 2005. Julio 13 de 2005. (Agosto 4 de 2006). Disponible en: http:// www.ins.gov.co/pdf/vcsp/informe_evento _ vih junio_1sem2005.pdf.

31. Ordóñez N, Castañeda E. Serotipificación de aislamientos clínicos y del medio ambiente de Cryptococcus neoformans en Colombia. Biomédica 1994;14:131-9.

32. Kwon-Chung KJ, Polacheck I, Bennett JE. Improved diagnostic medium for separation of Cryptococcus neoformans var. neoformans (serotypes $A$ and $D$ ) and Cryptococcus neoformans var. gattii (serotypes B and C). J Clin Microbiol 1982;15:535-7.

33. Gherna RL. Culture Preservation. En: P Gerhardt, RGE Murray, Willis A Wood, NR Krieg, eds. Methods for General and Molecular Bacteriology. Washington: American Society for Microbiology; 1994. p.278-92.

34. National Committee for Clinical Laboratory Standards. Reference method for broth dilution antifungal susceptibility testing of yeasts; approved Standard. M27-A, Vol 17, Wayne, Pennsylvania, USA NCCLS, 1997.

35. Meyer W, Castañeda A, Jackson S, Huynh M, Castañeda E; IberoAmerican Cryptococcal Study Group. Molecular typing of Ibero American Cryptococcus neoformans isolates. Emerg Infect Dis. 2003;9:189-95

36. Escandón $\mathbf{P}$, Sánchez A, Martínez $\mathbf{M}$, Meyer W, Castañeda E. Molecular epidemiology of clinical and environmental isolates of the Cryptococcus neoformans species complex reveals a high genetic diversity and the presence of the molecular type VGII mating type a in Colombia. FEMS Yeast Res 2006;6:625-35.

37. Kaufman L, Blumer S. Cryptococcosis: the awakening giant. En : Proceedings of the fourth international conference on the mycoses. The black and white yeast. PAHO Scientific Publication No. 354. 1978. Washington DC p.176-182.

38. Levitz S, Boekhout T. Cryptococcus: The once sleeping giant is fully awake. FEMS Yeast 2006; 6:461-2.

39. INS. Sistema Nacional de Vigilancia en Salud PublicaSIVIGILA. (Agosto 14 de 2006). Disponible en: http:// www.ins.gov.co/vigilancia/nivel2.php?seccion=28.

40. Oliveira JF, Greco DB, Oliveira GC, Christo PP, Guimaraes MD, Oliveira RC. Neurological disease in HIV-infected patients in the era of highly active antiretroviral treatment: a Brazilian experience. Rev Soc Bras Med Trop 2006;39:146-51.

41. Mirza SA, Pelan M, Rimland D, Graviss E, Hamill R, Brandt ME et al. The changing epidemiology of 
cryptococcosis: an update from population-based active surveillance in 2 large metropolitan areas, 1992-2000. Clin Infect Dis 2003;36:789-94.

42. FIMUA Cryptococcosis Network. European Confederation of Medical Mycology (ECMM) prospective survey of cryptococcosis: report from Italy. Med Mycol 2002;40:507-17.

43. Chen S, Sorrell T, Nimmo G, Speed B, Currie B, Ellis $\mathbf{D}$ et al. Epidemiology and host- and varietydependent characteristics of infection due to Cryptococcus neoformans in Australia and New Zealand. Australasian Cryptococcal Study Group. Clin Infect Dis 2000;31:499-508.

44. French N, Gray K, Watera C, Nakiyingi J, Lugada E, Moore $\mathbf{M}$ et al. Cryptococcal infection in a cohort of HIV-1 infected Ugandan adults. AIDS 2002;16:1031-8.

45. Rozenbaum R, Goncalvez AJ. Clinical epidemiological study of 171 cases of cryptococcosis. Clin Infect Dis 1994;18:369-80.

46. Correa MP, Oliveira EC, Duarte RR, Pardal PP, Oliveira $\mathbf{F}$ de $\mathbf{M}$, Severo LC. Criptococose em crianças no estado do Pará, Brasil. Rev Soc bras Med trop 1999;32:505-8.

47. Darze C, Lucena R, Gomes I, Melo A. Características clínicas laboratoriais de 104 casos de meningo-encefalite criptocócica. Rev Soc Bras Med Trop 2000;33:21-6.

48. Goldman DL, Khine H, Abadi J, Lindenberg DJ, Pirofski La, Niang $\mathbf{R}$ et al. Serologic evidence for Cryptococcus neoformans infection in early childhood. Pediatrics 2001;107:E66.

49. Kaur R, Mittal N, Rawat D, Mathur MD. Cryptococcal meningitis in a neonate. Scand J Infect Dis 2002; 34:542-3.

50. Gavai M, Gaur S, Frenkel LD. Successful treatment of cryptococcosis in a premature neonate. Pediatr Infect Dis J 1995;14:1009-10.

51. Shih CC, Chen YC, Chang SC, Luh KT, Hsieh WC. Cryptococcal meningitis in non-HIV infected patients. Q J M 2000;93:245-51.

52. Aberg JA, Mundy LM, Powderly WG. Pulmonary cryptococcosis in patients without HIV infection. Chest 1999;115:734-40.

53. Pappas PG, Perfect JR, Cloud GA, Larsen RA, Pankey GA, Lancaster DJ et al. Cryptococcosis in human immunodeficiency virus-negative patients in the era of effective azole therapy. Clin Infect Dis 2001;33:690-9.

54. Kontoyiannis DP, Peitsch WK, Reddy BT, Whimbey EE, Han XY, Bodey GB et al. Cryptococcosis in patients with cancer. Clin Infect Dis 2001;32:E145-50.

55. Chariyalertsak $\mathbf{S}$, Sirisanthana T, Saengwonloey $\mathbf{O}$, Nelson KE. Clinical presentation and risk behaviors of patients with acquired immunodeficiency syndrome in Thailand, 1994-1998: regional variation and temporal trends. Clin Infect Dis 2001;32:955-62.

56. Pappalardo MC, Melhem MS. Cryptococcosis: a review of the Brazilian experience for the disease. Rev Inst Med Trop Sao Paulo 2003;45:299-305.

57. Mwaba P, Mwansa J, Chintu C, Pobee J, Scarborough M, Portsmouth $S$ et al. Clinical presentation, natural history, and cumulative death rates of 230 adults with primary cryptococcal meningitis in Zambian AIDS patients treated under local conditions. Postgrad Med J 2001;77:769-73.

58. Graybill JR, Sobel J, Saag M, van Der Horst C, Powderly W, Cloud G et al. Diagnosis and management of increased intracranial pressure in patients with AIDS and cryptococcal meningitis. The NIAID Mycoses Study Group and AIDS Cooperative Treatment Groups. Clin Infect Dis 2000;30:47-54.

59. Lizarazo J. Medición de la presión de apertura del LCR durante la punción lumbar. Rev Fac Med Univ Nac Colomb 2006; 54: 66-7.

60. Shoham S, Cover C, Donegan N, Fulnecky E, Kumar P. Cryptococcus neoformans meningitis at 2 hospitals in Washington, D.C.: adherence of health care providers to published practice guidelines for the management of cryptococcal disease. Clin Infect Dis 2005;40:477-9.

61. Chang WC, Tzao C, Hsu HH, Lee SC, Huang KL, Tung HJ et al. Pulmonary cryptococcosis: comparison of clinical and radiographic characteristics in immunocompetent and immunocompromised patients. Chest 2006;129:333-40.

62. Popovich MJ, Arthur RH, Helmer E. CT of intracranial cryptococosis. Am J Neuroradiol 1990;11:139-42.

63. Perfect JR, Casadevall A. Cryptococcosis. Infect Dis Clin North Am 2002;16:837-74.

64. Feldmesser M, Harris C, Reichberg S, Khan S Casadevall A. Serum cryptococcal antigen in patients with AIDS. Clin Infect Dis 1996;23:827-30.

65. Sorrell TC. Cryptococcus neoformans variety gattii. Med Mycol 2001;39:155-68.

66. Meyer W, Marszewska K, Amirmostofian M, Igreja RP, Hardtke C, Methling $\mathrm{K}$ et al. Molecular typing of global isolates of Cryptococcus neoformans var. neoformans by polymerase chain reaction fingerprinting and randomly amplified polymorphic DNA -a pilot study to standardize techniques on which to base a detailed epidemiological survey. Electrophoresis 1999;20:1790-9.

67. Ellis D, Marriott D, Hajjeh RA, Warnock D, Meyer W, Barton R. Epidemiology: surveillance of fungal infections. Med Mycol 2000;38 (Suppl. 1):173-82.

68. Kidd SE, Hagen F, Tscharke RL, Huynh M, Bartlett $\mathrm{KH}$, Fyfe $\mathrm{M}$ et al. A rare genotype of Cryptococcus 
gattii caused the cryptococcosis outbreak on Vancouver Island (British Columbia, Canada). Proc Natl Acad Sci USA 2004;101:17258-63.

69. van der Horst CM, Saag MS, Cloud GA, Hamill RJ, Graybill JR, Sobel JD et al. Treatment of cryptococcal meningitis associated with the acquired immunodeficiency syndrome. National Institute of Allergy and Infectious Diseases Mycoses Study Group and AIDS Clinical Trials Group. N Eng J Med 1997;337:15-21.

70. Saag MS, Cloud GA, Graybill JR, Sobel JD, Tuazon CU, Johnson PC et al. A comparison of itraconazole versus fluconazole as maintenance therapy for AIDSassociated cryptococcal meningitis. National Institute of Allergy and Infectious Diseases Mycoses Study Group. Clin Infect Dis 1999;28:291-6.

71. Lizarazo J, Restrepo A, Castañeda E y el Grupo Colombiano de estudio de la criptococosis. Supervivencia y secuelas de pacientes registrados por el Grupo Colombiano de Estudio de la Criptococosis (1997-2001). Inf Quinc Epidemiol Nac 2002;7:449-53. 